\title{
Interstitial photoacoustic technique and computational simulation for temperature distribution and tissue optical properties in interstitial laser photothermal interaction
}

\author{
Zhifang Li*, Haiyu Chen ${ }^{\dagger}$, Feifan Zhou*, Hui Li ${ }^{*}, \S, \|$ and Wei R. Chen ${ }^{*}$, ,,$\|$ \\ ${ }^{*}$ College of Photonic and Electronic Engineering, Fujian Normal University \\ Fujian Provincial Key Laboratory of Photonic Technology \\ Key Laboratory of Optoelectronic Science and Technology for Medicine \\ Ministry of Education, Fuzhou, Fujian 350007, P. R. China \\ ${ }^{\dagger}$ Department of Cardiovascular Surgery, Fujian Provincial Hospital \\ Fujian Medical University, Fuzhou, Fujian 350000, P. R. China \\ *Bophotonics Research Laboratory \\ Center for Interdisciplinary Biomedical Education and Research \\ University of Central Oklahoma, Edmond, Oklahoma 73034, USA \\ \$hli@fjnu.edu.cn \\ 『wchen@uco.edu
}

Received 2 October 2016

Accepted 19 February 2017

Published 23 March 2017

\begin{abstract}
Interstitial laser immunotherapy (ILIT) is designed to use photothermal and immunological interactions for treatment of metastatic cancers. The photothermal effect is crucial in inducing anti-tumor immune responses in the host. Tissue temperature and tissue optical properties are important factors in this process. In this study, a device combining interstitial photoacoustic (PA) technique and interstitial laser photothermal interaction is proposed. Together with computational simulation, this device was designed to determine temperature distributions and tissue optical properties during laser treatment. Experiments were performed using ex-vivo porcine liver tissue. Our results demonstrated that interstitial PA signal amplitude was linearly dependent on tissue temperature in the temperature ranges of $20-60^{\circ} \mathrm{C}$, as well as $65-80^{\circ} \mathrm{C}$, with a different slope, due to the change of tissue optical properties. Using the directly measured temperature in the tissue around the interstitial optical fiber diffusion tip for calibration, the theoretical temperature distribution predicted by the bioheat equation was used to extract optical properties of tissue. Finally, the three-dimensional temperature distribution was simulated to guide tumor destruction and immunological stimulation. Thus, this novel device and method could be used for monitoring and controlling ILIT for cancer treatment.
\end{abstract}

Keywords: Photoacoustic imaging; photothermal effects; temperature.

${ }{ }_{\text {Corresponding authors. }}$

This is an Open Access article published by World Scientific Publishing Company. It is distributed under the terms of the Creative Commons Attribution 4.0 (CC-BY) License. Further distribution of this work is permitted, provided the original work is properly cited. 


\section{Introduction}

Laser immunotherapy $(\mathrm{LIT})^{1}$ was developed to treat late-stage, metastatic cancers through local laser irradiation and immunological stimulation. LIT has exhibited great potential both in preclinical stud$\operatorname{ies}^{2-6}$ and in preliminary clinical trials. ${ }^{7,8}$ Interstitial laser immunotherapy (ILIT) using an interstitial fiber with a cylindrical active diffusion tip could be an attractive alternative approach for deep-seated tumors and for patients with highly pigmented skins. In ILIT, the photothermal effect induced immune responses by destroying and interrupting tumor cells through temperature elevation in target tissue.

Most studies focused on the forward model of optical-thermal interaction between tissue and laser. ${ }^{9,10}$ And temperature distribution in biological tissues has been of interest in photothermal processes. Current noninvasive methods to measure tissue temperature include infrared thermography, ${ }^{11-13}$ ultrasound thermograph, ${ }^{14}$ and magnetic resonance thermometry (MRT). ${ }^{15-17}$ Infrared thermography could provide sensitive and real-time detection. However, it can only measure surface temperature. Ultrasound thermograph could reach deep tissues, but it had relatively low sensitivity and accuracy. And temperature measurement based on water proton resonance frequency $(\mathrm{PRF})$ in MRT has been used for interstitial laser therapy. ${ }^{15,16}$ MRT provided noninvasive three-dimensional temperature distribution with high sensitivity. However, it had relatively low temporal resolution and its cost and complexity severely limited its practical applications.

Recently, the PA technique has been used to measure optical properties and temperature distribution in biological tissues. Most of these studies have focused on temperature measurements with single-wavelength pulse laser excitation, without considering the effect of temperature changes on tissue properties. ${ }^{18,19}$ PA tomography has been applied for noninvasive temperature measurement, since the PA signal amplitude is linearly dependent on temperature in the range from $10^{\circ} \mathrm{C}-55^{\circ} \mathrm{C} .{ }^{18,20,21}$ In addition, a dual-wavelength $\mathrm{PA}$ technique has been developed to monitor tissue optical properties during thermal treatment, based on the observation that the ratio of PA signals generated by the two wavelengths is independent of temperature. ${ }^{23}$ However, most studies focus only on measured PA signals in the sample boundary ${ }^{24}$ with enhanced light absorption in target tissue. ${ }^{18}$ The PA response from tissues far from the boundary or without photoabsorbers often has low signal intensity. ${ }^{18}$

Since the photothermal effect depends on the tissue temperature and tissue optical properties, the temperature distribution and change of optical properties in tumors need to be simultaneously monitored, and the laser parameters need to be controlled accordingly, to optimize ILIT. In our study, we investigate the temperature distribution using PA signal and Pennes equation to provide a feedback to optical-thermal interactions. Interstitial PA imaging with computational simulation is used for simultaneous monitoring of temperature and optical properties during interstitial laser photothermal interaction. In this method, a continuouswave laser light and a pulsed laser light were delivered to the target area through the same optical fiber diffusion tip, for therapeutic photothermal irradiation and temperature measurement, respectively. During the interstitial laser photothermal interaction, the maximum temperature increase occurs in the tissue surrounding the cylindrical active fiber tip, as measured using the PA technique. In addition, the three-dimensional temperature distribution in tissue is simulated using the Pennes heat equation.

\section{Methods and Samples}

\subsection{Experimental setup}

The experimental setup for the combined PA detection and photothermal treatment system was shown in Fig. 1. The light of a pulsed Nd:YAG laser (Surelite I-10, Continuum, $810 \mathrm{~nm}$ ) with an OPO

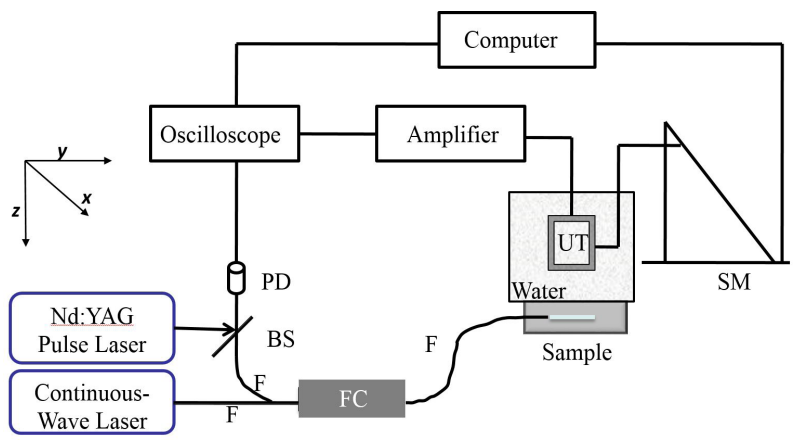

Fig. 1. Experimental setup. FC: Fiber coupler, F: Fiber, BS: Beam Splitter, PD: Photodiode, UT: focused ultrasound transducer, SM: Stepper motor. 
oscillator (Surelite OPO plus) and the light of a continuous-wave diode laser (ML-4030D, $810 \mathrm{~nm}$ ) were converged to a fiber coupler (FC) with a core diameter of $1000 \mu \mathrm{m}$ and delivered to the target tissue with an active cylindrical diffusion tip of $1.0 \mathrm{~cm}$.

The diode laser with a power of $10 \mathrm{~W}$ in this experiment was used to generate heat in target tissue. Interstitial irradiation by the diode laser lasted for a duration of $10 \mathrm{~min}$. The pulsed laser light was used to generate PA signals with a repetition frequency of $10 \mathrm{~Hz}$ and a pulse width of $6 \mathrm{~ns}$. The pulsed light beam was divided into two beams by a beam splitter (BS). One beam was received by a photodiode (PD) and displayed on the oscilloscope for calibration. The other beam was directed to the fiber coupler.

Porcine livers were procured from freshly slaughtered animals in a large slaughterhouse facility. And five samples were used for this experiment. The results were expressed by mean and standard deviation $(\mathrm{X} \pm \mathrm{SD})$. The size of the liver sample was $4 \mathrm{~cm} \times 4 \mathrm{~cm} \times 1 \mathrm{~cm}$. The sample was placed at room temperature $\left(22^{\circ} \mathrm{C}\right)$. The upper surface of the sample was coupled with a water bank by plastic wrap to reduce sound attenuation between different media (Fig. 1). The depth-resolved ( $z$ axial) photoacousitc signals from the sample were collected by a focused ultrasound transducer (UT) (V381, Panametrics) with a center frequency of 3.5 MHz. The transducer used has a bandwidth of $0-7 \mathrm{MHz}$, focal length at $3.5 \mathrm{MHz}$ of $6 \mathrm{~cm}$, diameter of $1.9 \mathrm{~cm}$, and focus zone of $3 \mathrm{~cm}$. In addition, the distance between the sample and transducer was $6 \mathrm{~cm}$. The signals were transmitted to an ultrasonic amplifier (5800 R, Parametric-NDT) and displayed on a digital oscilloscope (TDS3054C, Tektronix). A stepper motor (SM) (SC300-2B, Zolix) was used to accurately control the sample bracket by driving the electronic translation machine (TSA200-B, Zolix) for $2 \mathrm{D}$ scanning of the sample in the $x-y$ plane.

In order to improve scanning speed and signal-tonoise ratio (SNR), the signals on the digital oscilloscope were sampled without averaging and were analyzed using wavelet. $^{25}$ In our experimental setup, the pulsed laser light with a repetition frequency of $10 \mathrm{~Hz}$, and scanning step size of $0.1 \mathrm{~mm}$ were used to measure PA signal along the fiber tip of $1 \mathrm{~cm}$. It took $10 \mathrm{~s}$ to measure the PA signal along fiber tip of $1 \mathrm{~cm}$. The axial and lateral spatial resolution of PA imaging were $0.3 \mathrm{~mm}$ and $2 \mathrm{~mm}$, respectively. ${ }^{25}$

\subsection{Single integrating-sphere system for optical properties of tissue}

Tissue optical properties were measured by a single integrating-sphere system shown in Fig. 2. The single integrating-sphere system consisted of a laser (ML-4030D, $810 \mathrm{~nm}$ ), a light attenuator, a beam splitter, an optical power meter (Newport, 1918-R), an integrating sphere (Labsphere, Co. U.S.A.), and detectors. The diameter of the sphere was $152.4 \mathrm{~mm}$. The diameter of entrance and exit port of the integrating-sphere was $25.4 \mathrm{~mm}$, and Si photodiode. The laser beam passed through light attenuators and expanded into a collimated laser beam, the diameter of beam was $10 \mathrm{~mm}$. Then light beam passed through the beam splitter: one beam was detected by optical power meter and the other beam was perpendicular to the sample. The single integrating-sphere system shown in Fig. 2(a) was used for measuring the absorption coefficient. ${ }^{26}$ When the sample was placed in the exit port, the integrating-sphere was only used to measure the diffuse reflectance $R_{d}$ of the sample. When the sample was placed in the entrance port, the integrating sphere was only used to measure the diffuse transmittance $T_{d}$. The diffuse reflectance of the sample was given by $R_{d}=\left(\frac{K_{s}}{K_{p}}\right) R_{r}$, where $K_{p}$ is the diffuse reflection value of the standard target, $K_{s}$ is the diffuse reflection value of the sample, and $R_{r}$ is the reflection coefficient of the standard target. The diffuse transmittance of the sample could be expressed as $T_{d}=\frac{I_{s}}{I_{0}}$, where $I_{s}$ is the diffuse transmission value of the sample and $I_{0}$ is the total transmission value. The absorption coefficient $\mu_{a}$ of the sample was determined by using Kubelka-Munk theory ${ }^{26}$ based on the measurement of diffuse reflectance and transmittance.

$$
\mu_{a}=\frac{\sqrt{x^{2}-1}}{2(x+1) d} \ln \left[\frac{1-R_{d}(x-y)}{T_{d}}\right],
$$

where $x=1+R_{d}^{2}+T_{d}^{2} / 2 R_{d}, y=\sqrt{x^{2}-1}$ and, $d$ is the thickness of sample $(0.3 \mathrm{~mm})$. The attenuation coefficient can be measured by the single integratingsphere system as shown in the Fig. 2(b). In our experiment, the thickness of sample was 0.01, which was less than the mean free path. Based on Lambert-Beer law, attenuation coefficient was given by: $\mu_{t}=\mu_{a}+\mu_{s}=-\frac{1}{d_{c}} \ln \left(\frac{I_{c}}{I}\right)$, where $I_{c}$ is the collimated transmission value of the sample and $I$ was the initial light intensity. The scattering coefficient was $\mu_{s}=\mu_{t}-\mu_{a}$. 


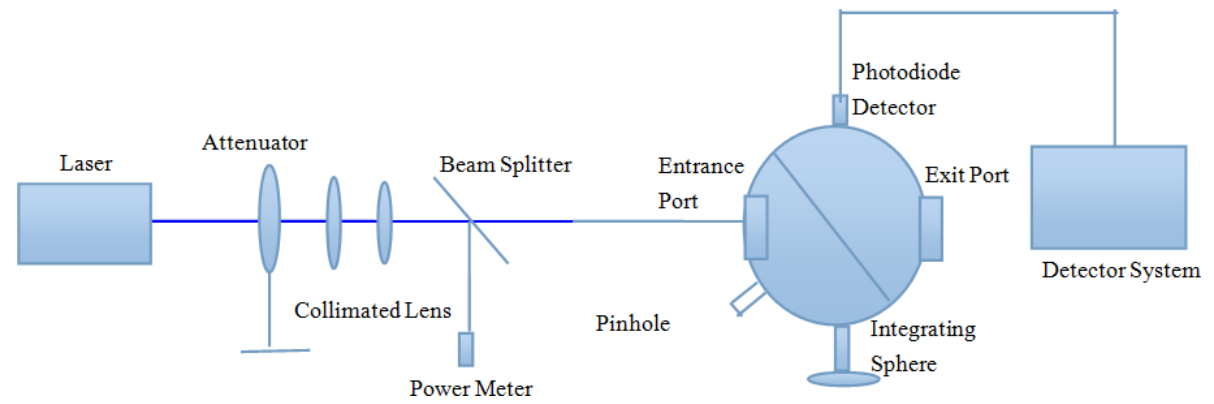

(a)

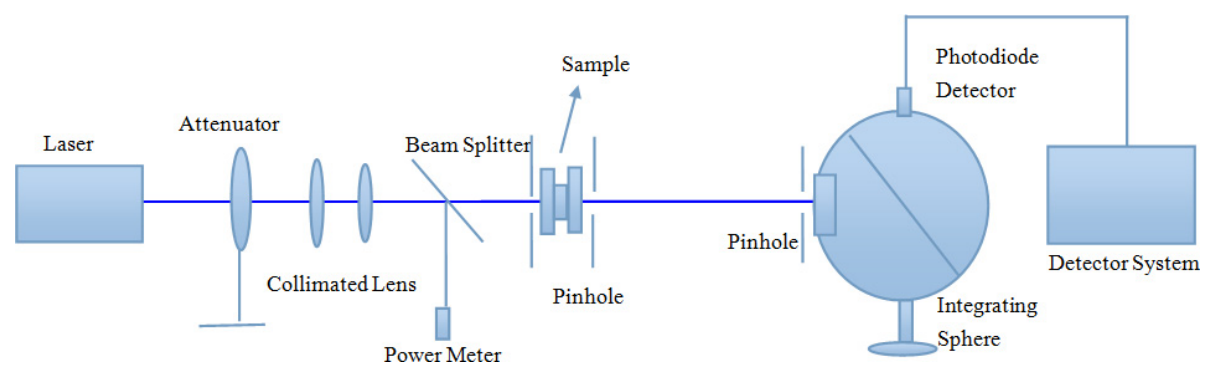

(b)

Fig. 2. Single integrating-sphere system for measurement of (a) absorption coefficient and (b) attenuation coefficient.

\subsection{Theoretical foundation of $P A$ measurement of tissue temperature}

PA detection used a short pulse laser to illuminate the absorbers in tissues to generate acoustic waves. The measured PA pressure that satisfies the temporal stress confinement is given by ${ }^{27}$

$$
P(z)=\Gamma \mu_{a} F\left(z, \mu_{a}, \mu_{s}, g\right),
$$

where $\Gamma$ is the Grüneisen parameter, $\mu_{a}$ is the absorption coefficient, $\mu_{s}$ is the scattering coefficient, $g$ is the anisotropic factor, $F\left(z, \mu_{a}, \mu_{s}, g\right)$ is the local optical fluence, and $z$ is the incident depth, as shown in Fig. 1.

During interstitial laser irradiation, both tissue temperature and tissue optical properties can change due to absorbed light energy. The parameter $\Gamma$ depends on both tissue temperature and tissue type/status. However, its average value showed no obvious differences whether the tissue is normal or coagulated. ${ }^{28}$ Thus, $\Gamma$ is only a function of temperature $T$ for most biological tissues.

\subsection{Simulation of tissue temperature distribution}

In our study, the continuous-wave laser light emitted from an interstitial diffusion tip was approximated as fourth degree polynomial function distributed along the tip. ${ }^{30}$ Photon propagation from the source was described by diffusion approximation ${ }^{9}$ :

$$
-D \nabla^{2} \phi(r)+\mu_{a} \phi(r)=s(r),
$$

where $\phi$ is the light power density $\left(\mathrm{W} \mathrm{cm}^{-2}\right), D$ is the diffusion coefficient $\left(\mathrm{cm}^{-1}\right), D=\left[3\left(\mu_{a}+\mu_{s}(1-g)\right]^{-1}\right.$, and $s$ is the source term $\left(\mathrm{W} \mathrm{cm}^{-3}\right)$.

The Pennes bioheat equation was used to simulate the temperature fields of tissue $\mathrm{e}^{10}$ :

$$
\begin{aligned}
\rho \cdot c & \frac{\partial T(r, t)}{\partial t} \\
= & \nabla \cdot[k \cdot \nabla T(r, t)]+\rho_{b} \cdot c_{b} \cdot \omega_{b} \cdot\left[T_{\text {art }}(r, t)\right. \\
& -T(r, t)]+S(r, t),
\end{aligned}
$$

where $T$ is the temperature $\left[{ }^{\circ} \mathrm{C}\right], \rho$ is the tissue density $\left[\mathrm{kg} \mathrm{cm}{ }^{-3}\right], c$ is the tissue specific heat $\left[\mathrm{J} \mathrm{kg}^{-1 \circ} \mathrm{C}^{-1}\right], k$ is the tissue thermal conductivity $\left[\mathrm{W} \mathrm{cm}{ }^{-1 \circ} \mathrm{C}^{-1}\right], r$ is the position vector $[\mathrm{cm}], t$ is the time $[\mathrm{s}], T_{\text {art }}$ is the temperature of arterial blood $\left[{ }^{\circ} \mathrm{C}\right], S$ is the deposited light power $\left[\mathrm{W} \mathrm{cm}^{-3}\right]$, and determined by $S=\mu_{a} \phi(r) \cdot \omega_{b}$ is the tissue average volumetric blood perfusion rate $\left[\mathrm{kg} \mathrm{s}^{-1} \mathrm{~cm}^{-3}\right], \rho_{b}$ is the density of blood, and $c_{b}$ is the specific heat of blood $\left[\mathrm{J} \mathrm{kg}^{-1} \mathrm{C}^{-1}\right]$. For an ex vivo tissue with no perfusion, $b$ can be assumed to be 0 . 
Table 1. Optical properties of porcine liver.

\begin{tabular}{lccc}
\hline Status & $\begin{array}{c}\text { Absorption } \\
\text { coefficient } \mu_{a}\end{array}$ & $\begin{array}{c}\text { Scattering } \\
\text { coefficient } \mu_{s}\end{array}$ & $\begin{array}{c}\text { Anisotropic } \\
\text { factor }(\mathrm{g})\end{array}$ \\
\hline Min value & $0.05 \mathrm{~mm}^{-1}$ & $5 \mathrm{~mm}^{-1}$ & 0.93 \\
Max value & $0.12 \mathrm{~mm}^{-1}$ & $39 \mathrm{~mm}^{-1}$ & 0.93 \\
\hline
\end{tabular}

Table 2. Thermal and physiological properties of porcine liver. ${ }^{9}$

\begin{tabular}{lllllll}
\hline$\rho$ & $c$ & $k$ & $A$ & $E_{a}$ & $R$ \\
\hline
\end{tabular}

Liver tissue $1.05 \quad 3.59 \quad 0.00566 \quad 7.39 \times 10^{39} \quad 2.577 \times 10^{5} \quad 8.314$

The thermal damage in tissue was described by damage parameter, $\Omega$, which is dimensionless, exponentially dependent on temperature, and linearly dependent on time of exposure. ${ }^{10}$ The interstitial laser ablation induced spatial-temporally dynamic optical properties of tissue, which depended on damage parameter $\Omega{ }^{10}$ Damage parameter $\Omega$ can be expressed as $\Omega(\mathbf{r}, t)=\int_{0}^{\tau} A e^{-E_{a} / R T(\mathbf{r}, t)} d t$, where $A\left[s^{-1}\right]$ is the frequency factor, $E_{a}[\mathrm{~J} / \mathrm{mole}]$ is the activation energy, $R\left[\mathrm{~J}\right.$ mole $\left.{ }^{-1} k^{-1}\right]$ is the universal gas constant, and $T[K]$ is the temperature. Damage $\Omega$ is dimensionless, exponentially dependent on temperature, and linearly dependent on time of exposure.

The temperature simulation was performed using Eqs. (2) and (3), with the tissue optical and thermal properties given in Tables 1 and 2 .

\subsection{Fitting optical properties of liver tissue during laser treatment}

To calibrate the PA technique for temperature determination, we used a thermocouple with a needle probe (core diameter $0.3 \mathrm{~mm}$ ) for direct temperature measurement. The needle probe was placed inside the target tissue at a distance of $0.8 \mathrm{~mm}$ from the center of the active cylindrical tip (Fig. 3). The detected superficial PA signal approximated the

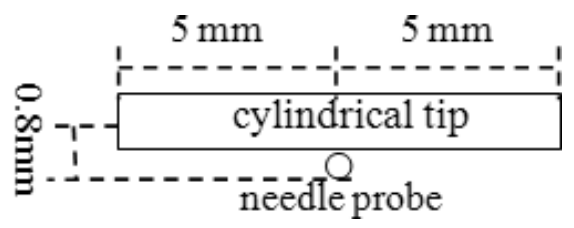

Fig. 3. The positions of the needle probe in relationship with the interstitial fiber tip. superposition of the PA signals from the depth of zero to $0.3 \mathrm{~mm}$, since the axial spatial resolution of PA imaging was $0.3 \mathrm{~mm}$. The temperature of tissue surrounding the fiber tip measured by the PA technique was the same as that measured by the thermocouple on the opposite side of the fiber tip due to symmetry. Therefore, the directly measured temperature could be used to calibrate the temperature measured by the PA transducer.

Optical properties $\left(\mu_{a}\right.$ and $\left.\mu_{s}\right)$ of the tissue during laser irradiation could be extracted by comparing temperature measurements by the PA technique inside the laser irradiated tissue with the simulated temperature distribution from an inverse heat transfer analysis (Eq. (3)) using COMSOL MULTIPHYSICS. In the simulation, the finite element method has been used to solve the light and bioheat transfer and their couple interactions. The minimum and maximum mesh sizes were $0.1 \mathrm{~mm}$ and $0.2 \mathrm{~mm}$, respectively, and time interval is $1 \mathrm{~s}$. In the light transfer simulation, initial value was of zero, and boundary condition satisfied Robin boundary. ${ }^{29}$ In bioheat transfer simulation, initial room temperature was set at $22^{\circ} \mathrm{C}$ and boundary was open. In the simulation, the values of the optical properties (Table 1) covered the range of the optical properties of native and coagulated liver tissue (Table 3) measured by single integration-sphere system. At the two extreme physiological conditions, the anisotropy factor for liver tissue remained the same, as shown in Table 3.

To extract dynamic values of the optical properties, we used an objective function based on the least square residual fit:

$$
\operatorname{LSQ}\left(\mu_{a}, \mu_{s}\right)=\frac{\sqrt{\sum_{i=1}^{M}\left[T_{p i}-T_{i}\left(\mu_{a}, \mu_{s}\right)\right]^{2}}}{M},
$$

where $M$ is the total number of measured temperatures along the diffusion fiber tip, $T_{p i}$ and $T_{i}$ represent the PA-measured temperature and simulated temperature, respectively, of the tissue in close vicinity of the diffusion fiber tip. During the temperature simulation, the values of the absorption and scattering coefficients were adjusted between that of native and coagulated status. The optical properties of the tissue under laser irradiation were determined by minimizing the objective function LSQ in Eq. (4). 


\section{Z. Li et al.}

Table 3. Comparison of the optical properties of liver determined by method in this study with single integration system.

\begin{tabular}{lllcc}
\hline Status & \multicolumn{1}{c}{ Method } & \multicolumn{1}{c}{$\begin{array}{c}\text { Absorption } \\
\text { coefficient } \mu_{a}\end{array}$} & $\begin{array}{c}\text { Scattering } \\
\text { coefficient } \mu_{s}\end{array}$ & $\begin{array}{c}\text { Anisotropic } \\
\text { factor }(\mathrm{g})\end{array}$ \\
\hline Native & Single integration system & $0.07 \pm 0.01 \mathrm{~mm}^{-1}$ & $6.1 \pm 0.07 \mathrm{~mm}^{-1}$ & $0.93 \pm 0.01$ \\
& Our method (photoacoustic) in this study & $0.063 \pm 0.006 \mathrm{~mm}^{-1}$ & $5.5 \pm 0.79 \mathrm{~mm}^{-1}$ & \\
Coagulated & Single integration system & $0.095 \pm 0.01 \mathrm{~mm}^{-1}$ & $13.3 \pm 0.1 \mathrm{~mm}^{-1}$ & $0.93 \pm 0.01$ \\
& Our method (photoacoustic) in this study & $0.075 \pm 0.008 \mathrm{~mm}^{-1}$ & $13.0 \pm 1.87 \mathrm{~mm}^{-1}$ \\
\hline
\end{tabular}

\section{Results}

\subsection{Correlation between temperature and PA signal}

During laser light exposure, temperatures close to the active fiber tip were measured by thermocouple and the PA amplitudes at a symmetric position, as shown in Fig. 4. The correlation curve in Fig. 4 showed three distinct regions. In Region I, the temperature was linearly proportional to PA amplitude from $20.2^{\circ} \mathrm{C}$ to $60.0^{\circ} \mathrm{C}$, with a fitting equation of: $T_{1}=1.1 \times$ PA-30.2. In Region III, the temperature was also linearly related to PA amplitude range from $65.0^{\circ} \mathrm{C}$ to $80.8^{\circ} \mathrm{C}$, with a fitting equation of: $T_{3}=2.6 \times \mathrm{PA}-179.6$. As shown in Fig. 4, the thermal process (the linearity of increase) in Regions I and II are noticeably different, indicating a change of thermal and optical properties of the tissue. Between $60^{\circ} \mathrm{C}$ to $65^{\circ} \mathrm{C}$ there was a transition region. In Region II, optical properties of liver tissue began to change. The results in Fig. 4

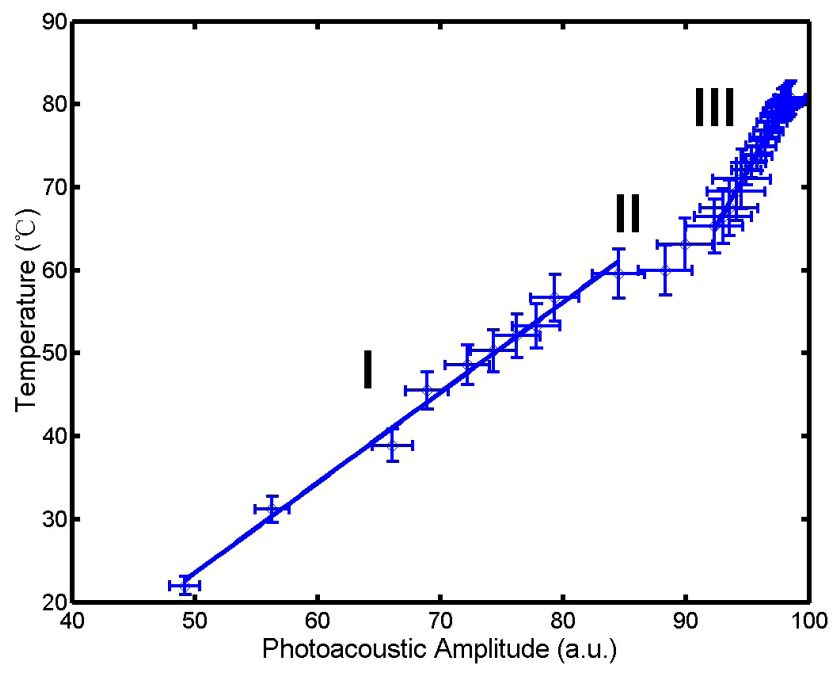

Fig. 4. Temperature versus PA amplitude during the tenminute exposure of continuous laser light. Temperature in terms of PA amplitude has three distinct parts. In parts I and III, the temperatures are linearly proportional to PA amplitude. Part II is the transition part. allowed us to determine tissue temperature based on PA signals.

\subsection{Temperature distribution by $P A$ measurement and simulation}

Figure 5 shows the temperature distributions in five tissue samples (four measurements for each sample) along the active fiber tip determined by PA signals immediately after laser irradiation and by simulation with least square residual fit. The objective function for the simulation using different absorption and scattering coefficients was given in Fig. 6 with the minimal objective function at $0.075 \mathrm{~mm}^{-1}$ and $13.0 \mathrm{~mm}^{-1}$, which were used for the simulation given in Fig. 5(d). From Table 3, the PA-measured optical properties were lower than integrating sphere results may be caused by two mainly reasons. One is that the thickness of sample was much smaller than the axial resolution of PA system, when the attenuation coefficient was measured by the integrating-sphere system. The other is that the lasers used were different in the PA system and in the integrating-sphere system. Although the wavelength of two laser were the same, the pulse laser was used in the PA system, and the continuous laser was employed in the integrating-sphere system. In addition, the diffusion equation used to describe the light transfer in tissue was more sensitive to diffusion coefficient $D$ than scattering coefficient and absorption coefficient.

\subsection{Three-dimensional temperature distribution by simulation}

With the absorption and scattering coefficients obtained using least square fitting (Fig. 6), the three-dimensional temperature distribution was simulated using Eq. (3). Figure 7 showed temperature distributions at cross-sections of different depths. The highest temperatures after laser irradiation were dependent on the distance from the active tip. 


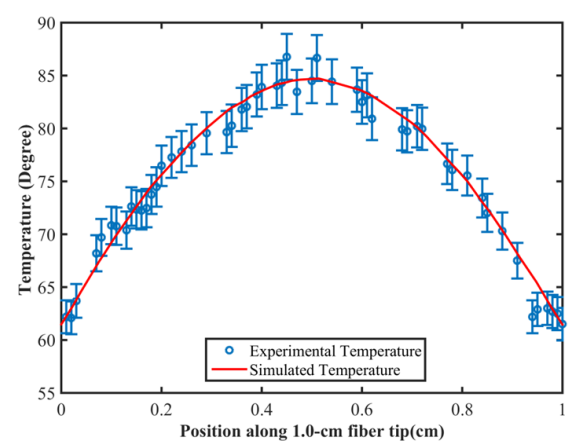

(a)

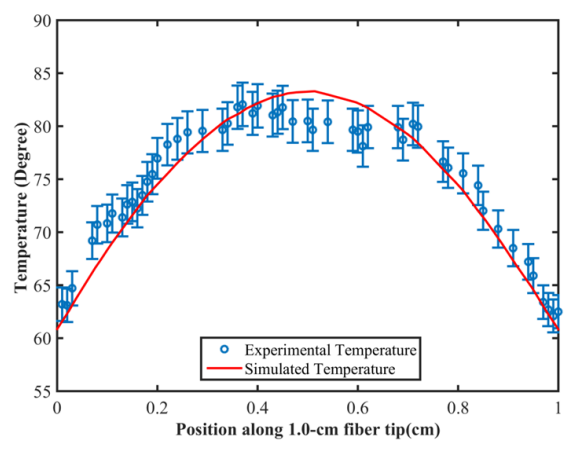

(d)

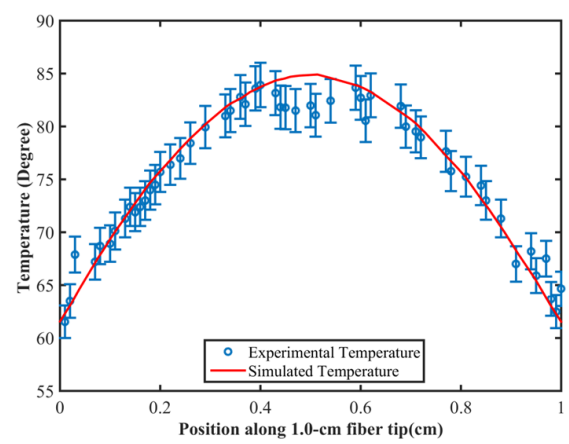

(b)

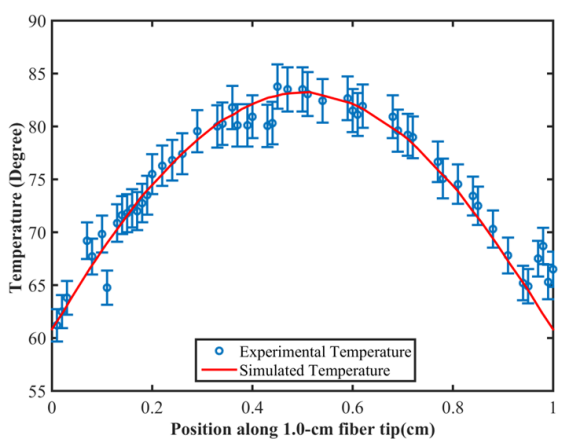

(c)

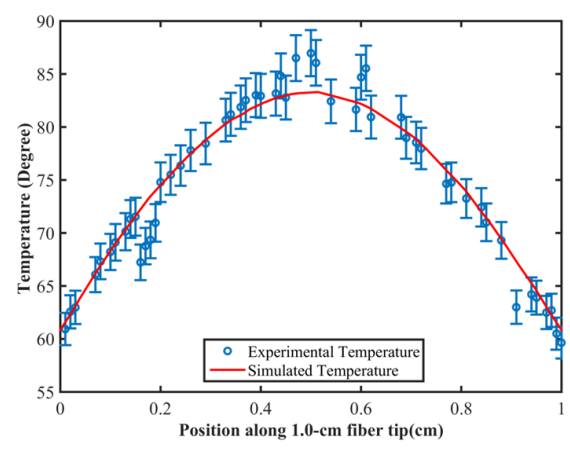

(e)

Fig. 5. Temperature distributions along fiber tip determined by PA signals (experimental) and computational simulation (theoretical) with (a) $\mu_{a}=0.07 \mathrm{~mm}^{-1}$ and $\mu_{s}=14.0 \mathrm{~mm}^{-1}$, (b) $\mu_{a}=0.08 \mathrm{~mm}^{-1}$ and $\mu_{s}=13.0 \mathrm{~mm}^{-1}$, (c) $\mu_{a}=0.065 \mathrm{~mm}^{-1}$ and $\mu_{s}=15.0 \mathrm{~mm}^{-1}$, (d) $\mu_{a}=0.075 \mathrm{~mm}^{-1}$ and $\mu_{s}=13.0 \mathrm{~mm}^{-1}$, (e) $\mu_{a}=0.085 \mathrm{~mm}^{-1}$ and $\mu_{s}=10.0 \mathrm{~mm}^{-1}$.

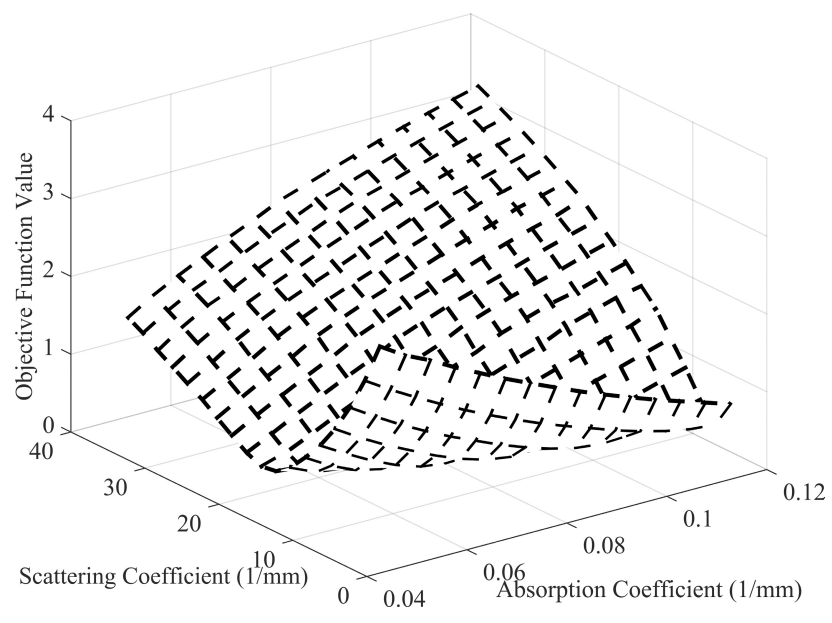

Fig. 6. The obtained objective function values for various scattering coefficients. The objective function is the smallest when $\mu_{a}=0.075 \mathrm{~mm}^{-1}$ and $\mu_{s}=13.0 \mathrm{~mm}^{-1}$.

\section{Discussions}

Gruneisen parameters $\Gamma$ depend on both the temperature and tissue type, but as indicated by authors in Ref. 28, the lack of a significant difference existed in Gruneisen parameters $\Gamma$ of native and coagulated tissue to tissue sample variability. For simplicity, we assume in the temperature range relevant to study, $\Gamma$ is independent to the tissue type. For a more accurate study, the tissue type, particularly the water contents of samples, should be considered.

Our simulation was able to extract the dynamic absorption and scattering coefficients of target tissue during laser photothermal interaction, as shown in Figs. 5 and 6. Furthermore, simulation can provide three-dimensional temperature maps.

At a temperature of $41-43^{\circ} \mathrm{C}$ or above, thermal interaction can induce different immunological responses. ${ }^{31}$ At a temperature of over $60^{\circ} \mathrm{C}$, tissue condensation, cell necrosis, and protein denaturation may occur. Therefore, temperature is an important factor in immunologically oriented phototherapy, such as ILIT. When ILIT is used for tumor treatment, target tissue temperature should be monitored and laser irradiation should be controlled for optimal effects.

This proposed PA-thermal coupling device is ideal for ILIT. Its novelty lies in its simplicity. The laser beam sources for treatment and temperature detection are uniquely coupled to irradiate the target tissue with spatial and temporal synchronization. 


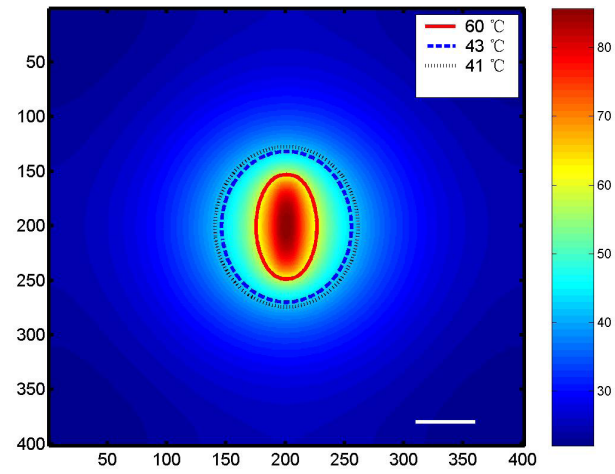

(a)

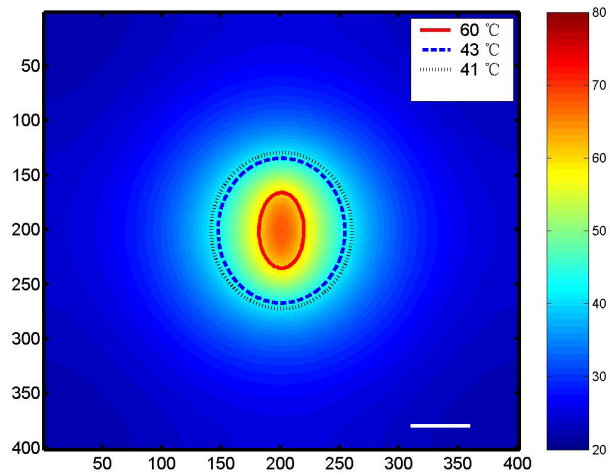

(c)

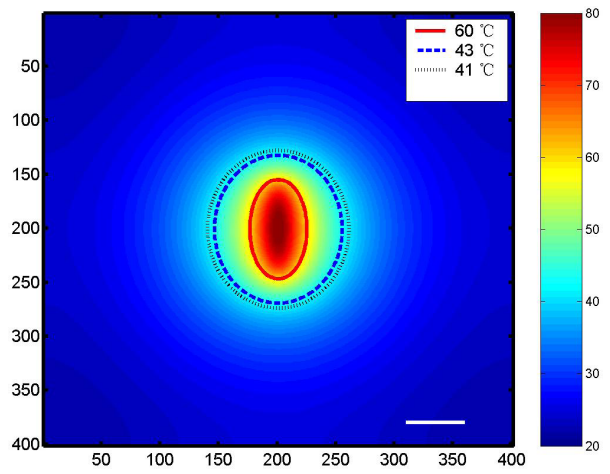

(b)

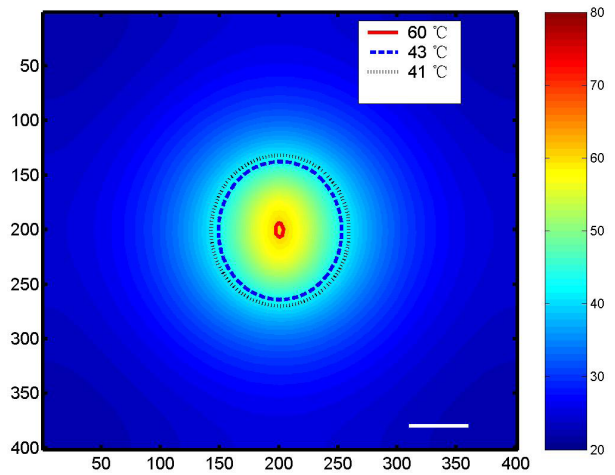

(d)

Fig. 7. (a) $x-y$ temperature image at different $z$ depths of (a) $0 \mathrm{~mm}$, (b) $0.5 \mathrm{~mm}$, (c) $1.5 \mathrm{~mm}$ and (d) $2.5 \mathrm{~mm}$. Scale bar $=5 \mathrm{~mm}$.

It is relatively easy and inexpensive to simultaneously obtain accurate tissue temperature and optical properties for real-time feedback and online control of laser photothermal therapy. The system can guide laser immunotherapy to achieve desirable thermal effects. Therefore, this technique has high clinical relevance. However, the measurement in this method was not real-time. In fact, we simulated the process and estimated the temperature and optical properties at least $60 \mathrm{~s}$ after laser light irradiation, since the tissue temperature increased sharply during the first $60 \mathrm{~s}$ and then slowly plateaued. ${ }^{32}$ In the future work, we will use a GPU to accelerate the postprocess and to increase the scanning speed of the array transducer to achieve a near real-time estimate.

It should be noted that an important characteristic of liver tissue is that it is richly perfused. Blood is the main absorber in the visible and near IR range of optical radiation and hemoglobin is known to transform at higher temperature into methemoglobin, which induces the change of optical properties of tissue. ${ }^{33-36}$ In this study, using ex vivo liver tissue, the dynamics of blood perfusion was not considered. In future studies, blood perfusion will be considered in the simulation and direct measurement in vivo will be used to calibrate the proposed device and method.

\section{Conclusion}

In this study, a special device was proposed to combine the interstitial PA technique and interstitial laser phototherapy for therapy and detection. Furthermore, combining PA detection and computational simulation, we developed a method to obtain the three-dimensional temperature distribution in target tissue during laser treatment. Our results demonstrate that the interstitial PA signal amplitude is dependent on tissue temperature in the range of $20-80^{\circ} \mathrm{C}$, with distinctive patterns. The correlation between temperature and PA signals can be used to determine tissue temperature in the range relevant to photothermal therapy. 


\section{Acknowledgments}

This project was sponsored in part by National Natural Science Foundation of China (No. 61675043/ 81571726), Fujian Provincial Natural Science Foundation (2015J01006), and by the US National Institutes of Health (R21 EB0155091). We also thank Janet Clark for proofreading the paper and for editorial assistance.

\section{References}

1. W. R. Chen, R. L. Adams, A. K. Higgins, K. E. Bartels, R. E. Nordquist, "Photothermal effects on murine mammary tumors using indocyanine green and an 808-nm diode laser: An in vivo efficacy study," Cancer Lett. 98, 169-173 (1996).

2. W. R. Chen, W.-G. Zhu, J. R. Dynlacht, H. Liu, R. E. Nordquist, "Long-term tumor resistance induced by laser photo-immunotherapy," Int. J. Cancer $\mathbf{8 1}$, 808-812 (1999).

3. W. R. Chen, H. Liu, J. A. Nordquist, R. E. Nordquist, "Tumor cell damage and leukocyte infiltration after laser immunotherapy treatment," Lasers Med. Sci. 15, 43-48 (2000).

4. W. R. Chen, A. K. Singhal, H. Liu, R. E. Nordquist, "Antitumor immunity induced by laser immunotherapy and its adoptive transfer," Cancer Res. 61, 459-461 (2001).

5. W. R. Chen, J. W. Ritchey, K. E. Bartels, H. Liu, R. E. Nordquist, "Effect of different components of laser immunotherapy in treatment of metastatic tumors in rats," Cancer Res. 62, 4295-4299 (2002).

6. W. R. Chen, S. W. Jeong, M. D. Lucroy, R. F. Wolf, E. W. Howard, H. Liu, R. E. Nordquist, "Induced anti-tumor immunity against DMBA-4 metastatic mammary tumors in rats using laser immunotherapy," Int. J. Cancer 107, 1053-1057 (2003).

7. X. Li, G. L. Ferrel, M. C. Guerra, T. Hode, J. A. Lunn, O. Adalsteinsson, R. E. Nordquist, H. Liu, W. R. Chen, "Preliminary safety and efficacy results of laser immunotherapy for the treatment of metastatic breast cancer patients," Photochem. Photobiol. Sci. 10, 817-821 (2011).

8. X. Li, M. F. Naylor, R. E. Nordquist, T. K. Teague, C. A. Howard, C. Murray, W. R. Chen, "In Situ Photoimmunotherapy for Late-stage Melanoma Patients: A Preliminary Study," Cancer Biol. Ther. 10(11), 1077-1214 (2010).

9. M. N. Iizuka, I. A. Vitkin, M. C. Kolios, M. D. Sherar, "The effect of dynamic optical properties during interstitial laser photocoagulation," Phys. Med. Biol. 45, 1335-1357 (2000).

10. Y. Mohammed, J. F. Verhey, "A finite element method model to simulate laser interstitial thermotherapy in anatomical inhomogeneous regions," Biomed. Eng. Online 4, 2 (2005).

11. A. J. Welch, M. J. C. van Gemert (Eds.), OpticalThermal Response of Laser-Irradiated Tissue, 1st edn. Plenum Publishing Corporation, New York (1995).

12. M. Mital, E. P. Scott, "Thermal detection of embedded tumors using infrared imaging," J. Biomech. Eng. 129(1), 33-39 (2007).

13. I. M. Gescheit, A. Dayan, M. Ben-David, I. Gannot, "Minimal-invasive thermal imaging of a malignant tumor: A simple model and algorithm," Med. Phys. 37(1), 211-216 (2010).

14. T. Varghese, J. A. Zagzebski, Q. Chen, U. Techavipoo, G. Frank, C. Johnson, A. Wright, F. T. Lee, "Ultrasound monitoring of temperature change during radofrequency ablation: Preliminary in vivo results." Ultrasound Med. Biol. 28(3), 321-329 (2002).

15. K. Le, X. Li, D. Figueroa, R. A. Towner, P. Garteiser, D. Saunders, N. Smith, H. Liu, T. Hode, R. E. Nordquist, W. R. Chen, "Assessment of thermal effects of interstitial laser phototherapy on mammary tumors using proton resonance frequency method," J. Biomed. Opt. 16(12), 128001 (2011).

16. Y. Chen, S. C. Gnyawali, F. Wu, H. Liu, Y. A. Tesiram, A. Abbott, R. A. Towner, W. R. Chen, "Magnetic resonance imaging guidance for laser photothermal therapy," J. Biomed. Opt. 13, 044033 (2008).

17. T. I. Oh, H. J. Kim, W. C. Jeong, M. Chauhan, O. I. Kwon, E. J. Woo, "Detection of temperature distribution via recovering electrical conductivity in MREIT," Phys. Med. Biol. 58(8), 2697-2711 (2013).

18. J. Shah, S. Park, S. Aglyamov, T. Larson, L. Ma, K. Sokolov, K. Johnston, T. Milner, S. Y. Emelianov, "Photoacoustic imaging and temperature measurement for photothermal cancer therapy," J. Biomed. Opt. 13, 034024 (2008).

19. H. Cui, X. Yang, "Real-time monitoring of highintensity focused ultrasound ablations with photoacoustic technique: An in vitro study," Med. Phys. 38(10), 5345-5350 (2011).

20. I. V. Larina, K. V. Larin, R. O. Esenaliev, "Real-time optoacoustic monitoring of temperature in tissues," J. Phys. D, Appl. Phys. 38, 2633-2639 (2005).

21. M. Pramanik, L. V. Wang, "Thermoacoustic and photoacoustic sensing of temperature," J. Biomed. Opt. 14, 054024 (2009).

22. J. Yao, H. Ke, S. Tai, Y. Zhou, L. V. Wang, "Absolute photoacoustic thermometry in deep tissue," Opt. Lett. 38, 5228-5231 (2013).

23. Y. Hsiao, X. Wang, C. X. Deng, "Dual-wavelength photoacoustic technique for monitoring tissue status during thermal treatments," J. Biomed. Opt. 18(6), 067003 (2013). 
24. M. S. Singh, H. Jiang, "Elastic property attributes to photoacoustic signals: An experimental phantom study," Opt. Lett. 39, 3970-3973 (2014).

25. Z. Li, H. Li, H. Chen, W. Xie, "In vivo determination of acute myocardial ischemia based on photoacoustic imaging with a focused transducer," $J$. Biomed. Opt. 16, 076011 (2011).

26. R. Molenaar, J. J. ten Bosch, J. R. Zijp, "Determination of Kubelka-Munk Scattering and absorption coefficients by diffuse illumination," Appl. Opt. 38, 2068-2077 (1999).

27. P. Shao, B. Cox, R. J. Zemp, "Estimating optical absorption, scattering and Grueneisen distributions with multiple-illumination," Appl. Opt. 50, 31453154 (2011).

28. B. Soroushian, W. M. Whelan, M. C. Kolios, "Dynamics of laser induced thermoelastic expansion of native and coagulated ex-vivo soft tissue samples and their optical and thermomechanical properties," Proc. SPIE 7899, $78990 Z$ (2011).

29. A. Wang, R. Lu, L. Xie, "Finite element modeling of light propagation in turbid media under illumination of a continuous-wave beam," Appl. Opt. 55, 95103 (2016).

30. W. Xie, Y. Liu, Z. Li, H. Li, "Reconstruction of 3D light distribution produced by cylindrical diffuser in deep tissues based on photoacoustic imaging," Chin . Opt. Lett. 12, 051702 (2014).

31. H. G. Zhang, K. Mehta, P. Cohen, C. Guha, "Hyperthermia on immune regulation: A temperature's story," Cancer Lett. 271, 191-204 (2008).

32. Z. Li, H. Chen, F. Zhou, H. Li, W. Chen, "Interstitial photoacoustic sensor for the measurement of tissue temperature during interstitial laser phototherapy," Sensors 15, 5583-5593 (2015).

33. J. K. Barton, G. Frangineas, H. Pummer, J. F. Black, "Cooperative phenomena in two-pulse, twocolor laser photocoagulation of cutaneous blood vessel," Photochem. Photobiol. 73, 642-650 (2001).

34. L. Cordone, A. Cupane, M. Leone, E. Vitrano, "Optical absorption spectra of deoxyhemoglobin and oxyhemoglobins in the temperature range 300$20 \mathrm{~K}$ relation with protein dynamics," Biophys. Chem. 24, 259-275 (1986).

35. W. A. Eaton, J. Hofrichter, "Polarized absorption and linear dichroism spectroscopy of hemoglobin," Methods Enzymol. 76, 175-261 (1981).

36. W. A. Eaton, L. K. Hanson, P. J. Stephens, J. C. Sutherland, J. B. R. Dunn, "Optical spectra of oxyand deoxyhemoglobin, J. Am. Chem. Sot. 100, 4991-5003 (1978). 